\title{
La gestión tributaria en cajas de ahorros como herramienta de control.
}

\section{Tax management in savings banks as a control tool.}

Mónica Patricia Mayorga Díaz. ${ }^{1}$, Leguisamo Rosado Erika Vanessa. ${ }^{2}$, María Alexandra López Paredes. ${ }^{3}$ María Teresa Espinosa Jaramillo. ${ }^{4}$

\section{DOI: https://doi.org/10.33262/visionariodigital.v4i2.1213}

\begin{abstract}
.
The savings banks in Ecuador have been created as a need for financing, especially in the rural sector, administered by the leaders of the communities which lack a specific regulatory entity and according to the idiosyncrasy of these organizations of the popular and solidarity economy, which has impeded their economic and social growth; control procedures allow organizations to establish a systematic order of transactions carried out by the company, due to the frequent discoveries of fraud in organizations, they help to examine whether there is compliance with processes, policies in the company, in The tax management these controls allow to identify the needs for improvements in the processes that help to work efficiently and effectively, for this the activities that give the results required by the organizations must be planned. Therefore, the objective of this research is to review the documentary and bibliographic analysis of the tax management models to trivialize the movements of the savings banks. For this investigation, a documentary review of the provisions issued by the
\end{abstract}

\footnotetext{
${ }^{1}$ Universidad Regional Autónoma de Los Andes, Ambato Ecuador, monikmayorgad@gmail.com, ORCID: 00000003-3071-236X

${ }^{2}$ Profesional independiente, Ambato Ecuador, erikadfm2013@hotmail.com

${ }^{3}$ Universidad Técnica de Ambato, Ambato Ecuador, ma.lopez@uta.edu.ec

${ }^{4}$ Universidad de Zulia, Zulia Venezuela, espinosa.auditores@gmail.com
} 
control agencies, bulletins, guides, and guidelines was used to proceed with the construction of the information.

Keywords: Savings bank, control procedures, tax management, analysis.

\section{Resumen.}

Las cajas de ahorro en Ecuador han sido constituidas como necesidad de financiamiento, en especial en el sector rural, administradas por los dirigentes de las comunidades las cuales carecen de un ente regulador específico y acorde a la idiosincrasia de estas organizaciones de la economía popular y solidaria, lo que ha impedido el crecimiento económico y social de éstas; los procedimientos de control permiten a las organizaciones establecer un orden sistemático de las transacciones que realiza la empresa, debido a los frecuentes descubrimientos de fraudes en las organizaciones, los mismos ayudan a examinar si existe un cumplimiento de los procesos, políticas en la empresa, en la gestión tributaria estos controles permiten identificar las necesidades de mejoras en los procesos que ayuden a trabajar de manera eficiente y efectiva, para esto se debe planificar las actividades que den los resultados requeridos por las organizaciones. Por consiguiente, el objetivo de esta investigación es revisar el análisis documental y bibliográfico de los modelos de gestión tributaria para trivializar a los movimientos de las cajas de ahorro. Para esta investigación se utilizó una revisión documental de las disposiciones emitidas por los organismos de control, boletines, guías, lineamientos, para proceder a la construcción de la información.

Palabras claves: Caja de ahorro, procedimientos de control, gestión tributaria, análisis

\section{Introducción.}

En los últimos tiempos, debido a las complicaciones que se presentan en las organizaciones como descubrimientos de fraude y corrupción, que han sido involucradas empresas internacionales; se ha consolidado la implementación del control interno en dichas instituciones en diferentes países. Se ha identificado que esto no es un tema solo para los contadores sino también es responsabilidad de los miembros de la cúpula administrativa de las diferentes actividades económicas de cualquier nación o corporación (Quinaluisa, Ponce, Muñoz, Ortega, \& Pérez, 2018).

El procedimiento de control interno está basado en políticas y técnicas que contienen un ambiente de control, que lo establece la administración afianzando fortalezas mediante el levantamiento de métodos, debido a lo cual son el principal soporte para llevar a cabo los cambios que con tanta urgencia se requieren para alcanzar y ratificar la eficiencia, efectividad, eficacia y economía en todos los procesos por lo cual está diseñado para dar un grado de seguridad en la realización de los objetivos establecidos, y así resolver sus necesidades. (Barona, 2012) (Acuña, 2013) (Vega de la Cruz, 2016). 
La gestión tributaria corresponde al organismo que permite la recaudación de tributos, comprende funciones, gestiones, determinando las obligaciones tributarias y así cumpliendo con las obligaciones tributarias llevando a cabo los compromisos con el estado; los elementos de la gestión tributaria están constituidos por la base imponible, cuota de gravamen, cuota y deudas tributarias las cuales son de gran ayuda debido a que así se pueden cumplir debido a que es la base esencial para el cumplimiento fiscal. (Escuela de Negocios y Dirección, 2015); (Barreix, 2016); (Encarna, 2016); (Escuela Pública Digital, 2016); (Onrubia, 2016); (Salazar, 2016); (Servicio de Rentas Internas, 2018); (Seco \& Muñoz, 2018).

Actualmente el modelo que el Ecuador dentro del Servicio de Rentas Internas (SRI), corresponde al modelo de tres brechas, el mismo que permite establecer una gestión correcta en la Administración Tributaria del país, generando procesos que miden la eficiencia y efectividad de los controles tributarios que debe realizar la institución para lograr un mayor cumplimento voluntario y veraz de las obligaciones tributarias por parte del contribuyente (Dirección Nacional de Planificación y Gestión Estratégica , 2016).

\section{Cajas de ahorros}

La crisis financiera internacional, conllevó a que las entidades de crédito presenten importantes restricciones en el acceso a los mercados de capitales, debido a que se intensificó la competencia por la captación del ahorro, lo que provocó la elevación del costo de los pasivos y por ende el aumento de la morosidad, consecuencias notorias que se encuentran en las cajas de ahorro de España, ante lo cual se ha desarrollado una redefinición de sus estrategias y políticas, entre las cuales se encuentran los proceso de fusión y otras fórmulas posibles entre las que cabe citar los sistemas. Las cajas de ahorro surgieron a finales del siglo XVIII en Alemania e Inglaterra, en sus orígenes se utilizaron como instrumento de mejora de las condiciones de vida de las clases trabajadoras, a través de la remuneración del ahorro, las mismas que tuvieron como propósito proteger a la población que no tenía acceso al crédito o a los pobres con la finalidad de formar un capital con bienes de la comunidad y aportes de la misma población desprotegida. (Mayorga, Álvarez, Mayorga, \& Herrera, 2018). (Mayorga D. M., 2017)

En Ecuador las cajas de ahorro han sido constituidas como necesidad del financiamiento en especial a nivel rural, se constituyen con contribuciones económicos de sus socios, en calidad de ahorros, sin que puedan atraer fondos de terceros, asimismo, que su finalidad es de dar créditos a sus miembros, bajo los reglamentos que manifieste la Junta de Política y Regulación Monetaria y Financiera (JPRMF) y la supervisión que realice la Superintendencia de Economía Popular y Solidaria (SEPS)" (Ministerio de Economía y Finanzas, 2018). Existen fundaciones e instituciones que están ayudando a su fortalecimiento, mediante la capacitación, asistencia técnica y tecnología respectiva como la Red Nacional de Finanzas Populares y Solidarias del Ecuador, (RENAFIPSE), el Fondo Ecuatoriano Populorum Progressio (FEPP), el Consejo de Desarrollo de las Naciones y Pueblos del Ecuador (CODENPE), hoy la Unión de Cajas y Bancos Comunales de las 
Nacionalidades y Pueblos del Ecuador (UCABANPE), que nos permiten a visualizar en forma general su aporte, en la economía rural. (Junta de Regulación Monetaria y Financiera, 2018).

Tungurahua es la tercera provincia con mayor movimiento económico y comercial del Ecuador lo que da apertura de un incremento de cooperativas de ahorro y crédito, existiéndose a un total de ciento diecisiete de ellas. Las cajas de ahorro es una modalidad que existe en la provincia las cuales son administradas por dirigentes de comunidades, las mismas que facilitan créditos hasta ochocientos dólares, estas entidades no son reguladas por ninguna entidad o ente estatal, sino únicamente por miembros de las zonas en las que se desarrollan (Saltos, Mayorga, \& ruso, 2016). De cara al contexto empresarial es necesario analizar el marco regulador vigente en el Ecuador con relación al tema de estudio, que permita la operacionalización de las aristas de la investigación (tabla 1).

Tabla 1.- Marco regulador vigente

\section{Marco Jurídico}

Normativa Legal Vigente Extracto

(Constituyente, A., 2008) Art. 309: "El sistema financiero nacional se compone de los sectores público, privado,

y del popular y solidario, que intermedian recursos del público. Cada uno de estos Registro Oficial $N^{\circ} 449$ del 20 sectores contarán con normas y entidades de control específicas y diferenciada" Art. de octubre del 2008. 312.- "El sector financiero popular y solidario se compondrá de cooperativas de ahorro y crédito, entidades asociativas o solidarias, cajas y bancos comunales y cajas de ahorro" (Constituyente, A., 2008)

Art. 163 "Sector financiero popular y solidario: El sector financiero popular y solidario está compuesto por: 1. Cooperativas de ahorro

y crédito; 2. Cajas centrales; 3. Entidades asociativas o solidarias, cajas y bancos comunales y cajas de ahorro" (Código Monetario y Financiero, 2014).

Art 458: "Las cajas y bancos comunales y cajas de ahorro. Las entidades asociativas o solidarias, cajas y bancos comunales y cajas de ahorro son organizaciones que podrán optar por la personalidad jurídica, que se forman por voluntad de sus socios dentro del límite y en la forma determinada por la Junta de Política y Regulación Monetaria y Financiera, con propia estructura de gobierno, administración, representación, auto control social y rendición de cuentas y tendrán que remitir la información a la superintendencia” (Código Monetario y Financiero , 2014).

Art 459.-Legislación Aplicable: "Las entidades asociativas o solidarias, cajas y $\mathrm{y}_{\text {bancos comunales y cajas de ahorro se regirán por este Código, por la Ley Orgánica }}$ Oficial de la Economía Popular y Solidaria y las normas que expida la Junta de Política y Regulación Monetaria y Financiera. (Código Monetario y Financiero, 2014).

$\mathrm{N}^{\circ} 332$ del día viernes 12 de septiembre del año 
2014.

(Ley de Régimen Tributario Art. 98.- Definición de sociedad. - Para efectos de esta Ley el término sociedad Interno, 2018) comprende la persona jurídica; la sociedad de hecho; el fideicomiso mercantil y los patrimonios independientes o autónomos dotados o no de personería jurídica, salvo los constituidos por las Instituciones del Estado siempre y cuando los beneficiarios sean dichas instituciones..." (Ley de Régimen Tributario Interno, 2018).

(Ley Orgánica de Economía Art. 78 "Sector Financiero Popular y Solidario: Para efectos de la presente Ley, Popular y Solidaria, 2019) integran el Sector Financiero Popular y Solidario las cooperativas de ahorro y crédito, entidades asociativas o solidarias, cajas y bancos comunales, y cajas de ahorro" (Ley Registro Oficial Orgánica de Economía Popular y Solidaria, 2019)

$\mathrm{N}^{\circ} 444$ del 10 de

Mayo del año 2011

Art. 104.- "Entidades asociativas o solidarias, cajas y bancos comunales y cajas de ahorro. - Son organizaciones por voluntad de sus socios y con aportes económicos que, en calidad de ahorros, sirven para el otorgamiento de créditos a sus miembros" (Ley Orgánica de Economía Popular y Solidaria, 2019)

Art. 90.- "Entidades Asociativas o solidarias, cajas y bancos comunales. -Son organizaciones que pertenecen al Sector Financiero Popular y Solidario, que realizan sus actividades, exclusivamente, en los recintos, comunidades, barrios o localidades en donde se constituyen y se pueden financiar, con sus propios recursos o con fondos provenientes de programas o proyectos ligados al desarrollo de sus integrantes" (Reglamento a la Ley Orgánica de Economía Popular y Solidaria, 2012)

(Reglamento a la Ley Orgánica Art. 91.- "Cajas de Ahorro. -Son las organizaciones integradas por miembros de un de Economía Popular y mismo gremio o institución; por grupos de trabajadores con un empleador común, Solidaria, 2012) Registro grupos familiares, barriales; o, por socios de cooperativas distintas a las de ahorro y Oficial crédito". (Reglamento a la Ley Orgánica de Economía Popular y Solidaria, 2012)

$\mathrm{N}^{\circ} 648$ del 27 de

Febrero del año

2012
Art. 92.- "Constitución, organización y funcionamiento. - La constitución y organización de las entidades asociativas o solidarias, cajas y bancos comunales y cajas de ahorro, se regirá por lo dispuesto para las organizaciones comunitarias.” (Reglamento a la Ley Orgánica de Economía Popular y Solidaria, 2012)

Elaboración propia a partir de (Mayorga, 2018).

\section{Metodologia.}

La revisión documental consiste en buscar, seleccionar, organizar y analizar un conjunto de materiales escritos para responder una o varias preguntas sobre un tema, estableciendo semejanzas y diferencias entre los trabajos y las ideas del investigador, categorizar experiencias, distinguir los elementos más abordados con sus esquemas observacionales, y precisar ámbitos no explorados, colaborando con la fundamentación en la indagación y utilización de fuentes fidedignas en bases de datos reconocidas. (Valencia, 2015); (Bermeo, 2016); (Rodríguez D., Abella, \& Pineda C., 2016). 
Vol. 4, $\mathrm{N}^{\circ}$ 2, p. 22-39, abril - junio, 2020

En tal sentido, se revisaron los modelos de gestión tributaria que se acerquen a la realidad económica, social y ambiental de las cajas de ahorro, las mismas que se han considerado unidades de análisis para el desarrollo del presente estudio, así como también se efectúa la revisión documental de los reportes socializados por el organismo de control referentes a la organización y cumplimiento de obligaciones tributarias por parte de los niveles directivos de las organizaciones en estudio o sus representantes legales.

\section{Modelo de Gestión Tributaria}

El modelo de gestión tributaria en Ecuador consta de registros la presentación y pago de impuestos los cuales son dispuestos por el ente regulador de impuestos en el país el Servicio de Rentas Internas (SRI) proporcionando un control formal, material y extensivo sobre las cajas de ahorro (Figura 1).

Figura 1. Modelo de gestión tributaria

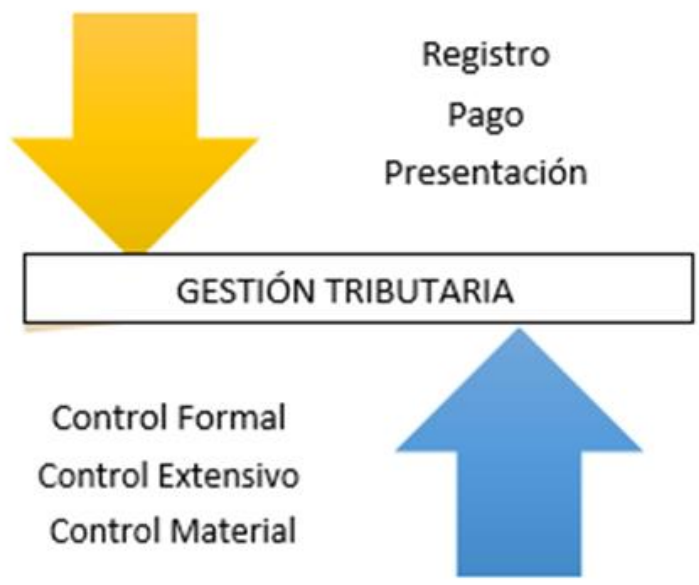

Elaboración propia a partir de (Servicio de Rentas Internas, 2018).

La Constitución del Ecuador en conjunto con el Plan Nacional de Desarrollo 2017-2021 Toda una vida, establece la construcción de un sistema tributario que promueva una redistribución solidaria y equitativa de la riqueza para todos los ecuatorianos, la misma que hace énfasis en la reducción de la pobreza y la desigualdad social. Para lograr el objetivo planteado, el Estado ecuatoriano debe avanzar hacia un sistema tributario con un enfoque progresivo mediante el cual los impuestos directos logren una mayor recaudación que los impuestos indirectos, ya que estos últimos no distinguen la capacidad económica del individuo (Dirección Nacional de Planificación y Gestión Estratégica, 2016).

En su planificación estratégica el Servicio de Rentas Internas propone un modelo de gestión y medición; el cual se conoce como el modelo de Brechas; en las que existen tres tipos de brechas que corresponden a la brecha de inscripción donde toda persona natural o jurídica debe registrase en dicho sistema; la brecha de presentación en la cual dicha brecha detalla los programas de 
controles como es la presentación de los Impuesto al Valor Agregado y el Impuesto a la Renta; este último se omite en las cajas de ahorros, debido que estas organizaciones deben invertir sus utilidades en su propia organización u obra social y por último la brecha de pago, en la cual deben realizar el pago de las obligaciones tributarias (Figura 2).

Figura 2. Modelo de Gestión Tributaria - Modelo de Brechas
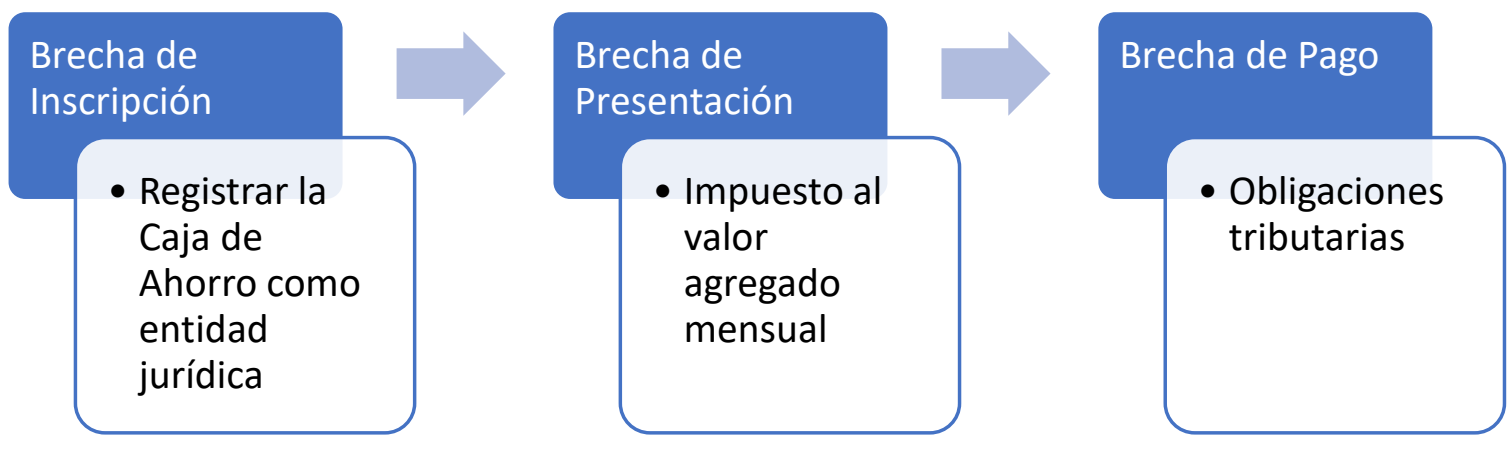

Elaboración propia a partir de (Servicios de Rentas Internas, 2016)

\section{Modelo de Consistencia}

Este modelo comprende en agrupar información en forma ordenada entre el balance de ingresos (gastos) y las transacciones financieras de los sectores institucionales; para el estudio de campo en las organizaciones analizadas, se lo debe realizar los ingresos de los socios a la caja de ahorro y las salidas del dinero mediante los créditos. (Tabla 2.)

Tabla 2. Modelo de Consistencia

\begin{tabular}{|c|c|l|l|l|l|}
\hline \multicolumn{5}{|c|}{ Modelo de Consistencias } \\
\cline { 2 - 6 } & Fórmula del Problema & Objetivos & Hipótesis & Variables & Controles \\
\hline 1 & Captación de Socios & & & & \\
\hline 2 & Montos de Créditos & & & & \\
\hline 3 & Inversiones & & & & \\
\hline 4 & Importaciones & & & & \\
\hline 5 & Intereses & & & & \\
\hline
\end{tabular}

Elaboración Propia a partir (Ramírez, 2010) 
En el modelo de consistencias se reflejan cinco cuadrantes donde se agrupará en forma ordenada las diferentes problemáticas de la caja de ahorro se determina dentro de este modelo : 1. Fórmula de problema: Se detallará en forma secuencial los diferentes enigmas que se encuentre dentro de la caja de ahorros y los socios, 2. Objetivo: En cada una de los cuadrantes se tendrá un objetivo para saber a qué se debe dirigir las fuerzas del análisis, 3. Hipótesis: Habrá una variable de hipótesis para confirmar una verdad, 4. Variable: dentro de este cuadrante se podrá medir; por ejemplo, el número de socios dividido par el número de créditos, 5.Controles: Se creará un sistema de control mediante las auditorías que se disponga dentro de la caja de ahorros.

\section{Modelo de Insumos Modelo Leóntiev o Tabla Input-output}

Wassily Leóntiev (1905 - 1999) fue un economista estadounidense de origen ruso; ganó el premio Nobel de economía en el año de 1973; representa uno de los sistemas de la actividad económica de una región, la palabra inglesa output se refiere al producto que sale de una empresa o industria, mientras que imput se refiere a los factores o recursos que se necesita para realizar esa producción total de cada sector productivo. (Figura 3.)

Figura 3. Modelo de Insumos

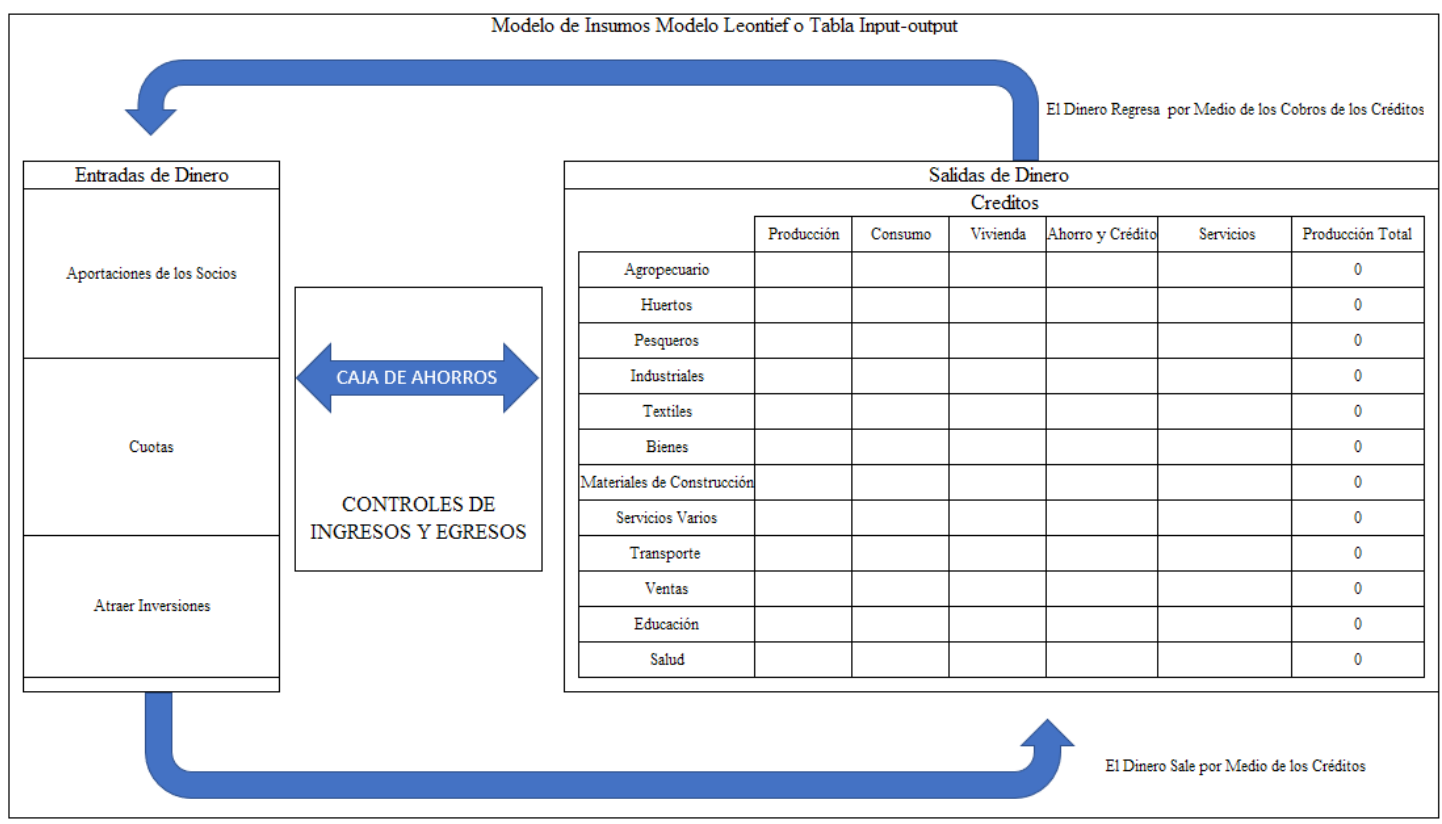

Elaboración Propia a partir de (Ramírez, 2010)

Este modelo de Insumos Modelo Leóntiev o Tabla Input-output que se utilizará en nuestro sistema de cajas de ahorro mediante una relación estrecha entre las entradas y salidas del dinero (Figura 3).

Se realizará un control de los dineros mediante auditorias de los ingresos y los egresos. 
- Entradas de Dinero: mediante la captación de los socios

- Salidas de Dinero: mediante el uso de los créditos a los socios en los diferentes servicios y productos que ofrece la caja de ahorros

- Los controles se realizarán mediante los balances diarios donde se alimentará diariamente el sistema para tener una información oportuna, real y permanente.

\section{Modelo de Equilibrio General}

Este modelo tiene la capacidad de detener todas las transferencias de la economía, se proyecta mediante el comportamiento racional de los hogares y las empresas dentro un solo mercado pequeño y competitivo, considerando invariable al resto de la economía, se utiliza para anticipar posibles problemas en el futuro, facilitando en el día a día las soluciones mediante en el estudio de las interacciones. (Figura 4.)

Figura 4. Modelo de Equilibrio General

MODELO DE EQUILIBRIO

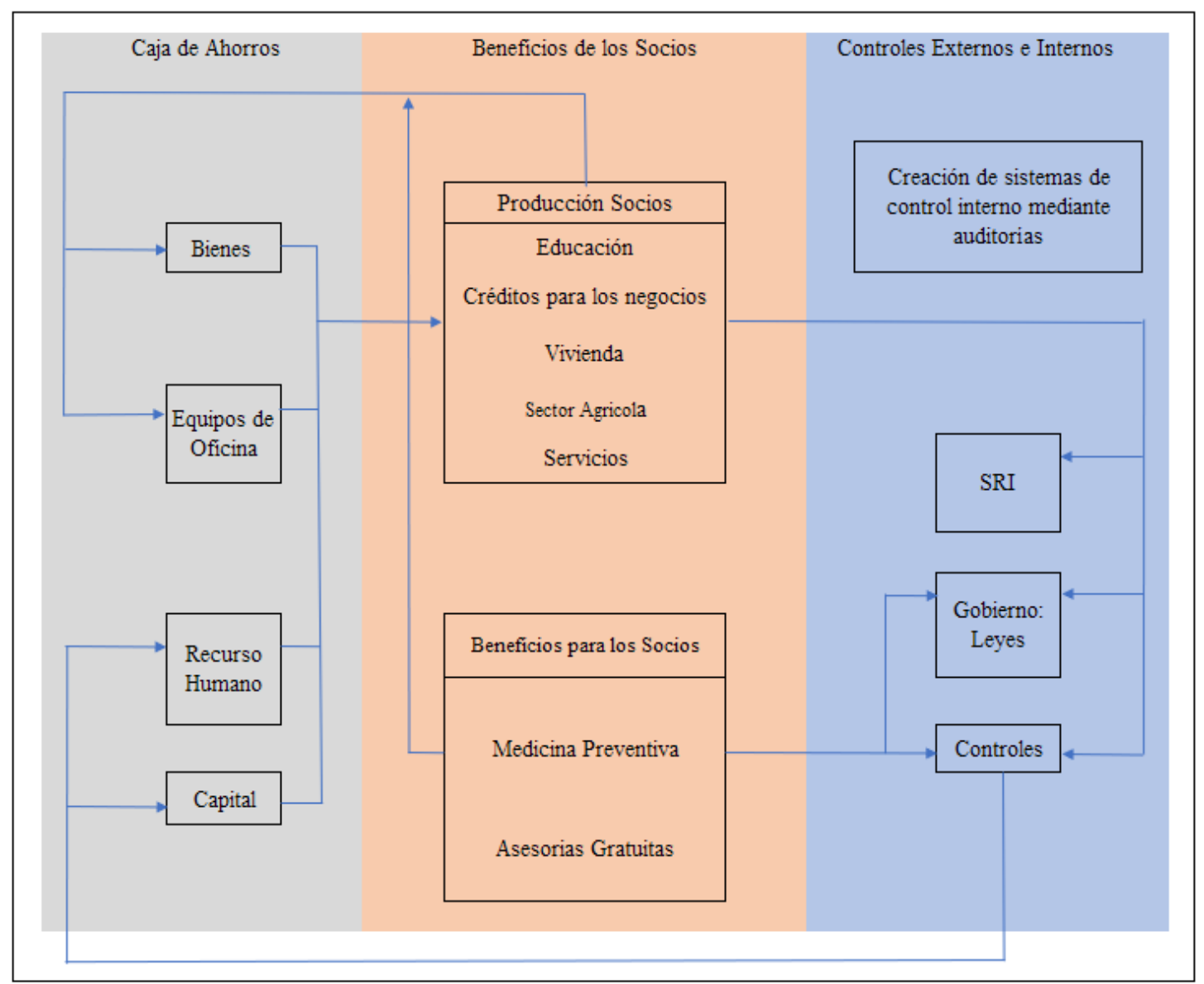

Elaboración Propia a partir de (Ramírez, 2010) 
Este modelo de Equilibrio está basado en las necesidades de los socios y tiene tres cuadrantes (Figura 4), los cuales detallaremos a continuación:

- $\quad$ Patrimonio de la caja de ahorro: Se encuentra segmentada en cuatro niveles.

- Bienes: Son todos los inmuebles que debe tener la caja de ahorros pudiendo ser estos: el terreno, la construcción, patio automotor; etc., para el desenvolvimiento y funcionabilidad de los socios.

- Equipos de Oficia: Contempla todos los enseres dentro de las oficinas, como son: computadoras, muebles, papelería, etc.

- $\quad$ Recurso Humano: el personal calificado para el desarrollo de las actividades diarias.

- Capital: será el recurso de las aportaciones de los socios

- $\quad$ Beneficios de los socios: Como su palabra lo indica será el provecho que obtendrán los socios de acuerdo a las necesidades que estos tengan.

- $\quad$ Producción Socios: Este cuadrante está constituido para la parte de la producción donde los socios obtendrán sus créditos para implementar sus negocios, para comprar terrenos o casas, para el segmento agrícola como semillas, fertilizantes, o pagos de servicios que requieran.

- Dentro del cuadrante se tendrá los beneficios como es la salud y el asesoramiento para las diferentes actividades que requiera los socios.

- Controles Externos e Internos: Dentro de este cuadrante se observa las diferentes obligaciones con los socios y el estado, para ello se realizará las diferentes inspecciones, supervisiones para el desarrollo de las actividades económicas.

- $\quad$ SRI: pagos mensuales de las responsabilidades con el estado.

- Gobierno y Leyes: Conocer los estatutos que dictamine el gobierno mediante sus normativas

- Controles: serán las auditorías

Dentro de las cuales se observan líneas de correlación que existe entre estas; por ejemplo, si no se tiene dentro de la caja de ahorro el terreno, las oficinas, un personal calificado y el capital necesario no se podría trabajar con las necesidades de los socios y entregar sus beneficios, aparte de ello se requiere de los diferentes controles y pagos de impuestos para el desarrollo de las cajas de ahorro como se aprecia con las líneas de color azul.

\section{Resultados.}

Para el efecto del estudio se va a tomar el modelo de Brechas donde se podrá consolidar las cajas de ahorro como persona jurídica y establecer una estructura interna, la cual permite formar los cimientos para en el futuro, de acuerdo a los ingresos de los socios se pueda convertir en una cooperativa de ahorro y crédito consolidándose en el sistema financiero como una entidad de mayor capacidad; además de generar un control al declarar las obligaciones tributarias.

De cara a la realidad de la gestión tributaria y el control en las cajas de ahorro; se considera la aplicación del modelo de brechas en razón de que al aplicar este modelo, las estructuras internas deberán generar procedimientos de control interno que permitan identificar la gestión tributaria 
realizada por las cajas de ahorros; un modelo de control que permita establecer una metodología de diagnóstico y evaluación GRECC: Gobierno Cooperativo-Asociativo(G), Riesgos (R), Evaluación Económico-Financiero (E), Control Interno (C), Calidad, que ayude a la Superintendencia de Economía Popular y Solidaria a controlar, por medio de los auditores calificados, dichas organizaciones (Hernández, 2018).

Dentro del control en las cajas de ahorro, el Reglamento a la Ley Orgánica de Economía Popular y Solidaria del sistema financiero en su artículo 107 estipula que las instituciones controladas por la Superintendencia "en sus operaciones de crédito, deberán sujetarse a las tasas de interés fijadas por el Banco Central del Ecuador y deberán cumplir con las normas de prevención de lavado de activos que determine la ley"; además de "contratar auditoría externa anual y auditoría interna" de acuerdo a las disposiciones de la Junta de Regulación; para esto la Superintendencia califica a los auditores, quienes deberán desarrollar su actividad profesional de acuerdo al cumplimiento a la Ley, por lo que es necesario efectuar la revisión documental a los reportes de control efectuados por el SRI a estas organizaciones. (Tabla 3.)

Tabla 3.- Revisión documental de procedimientos de control en cajas de ahorro

\section{Revisión documental}

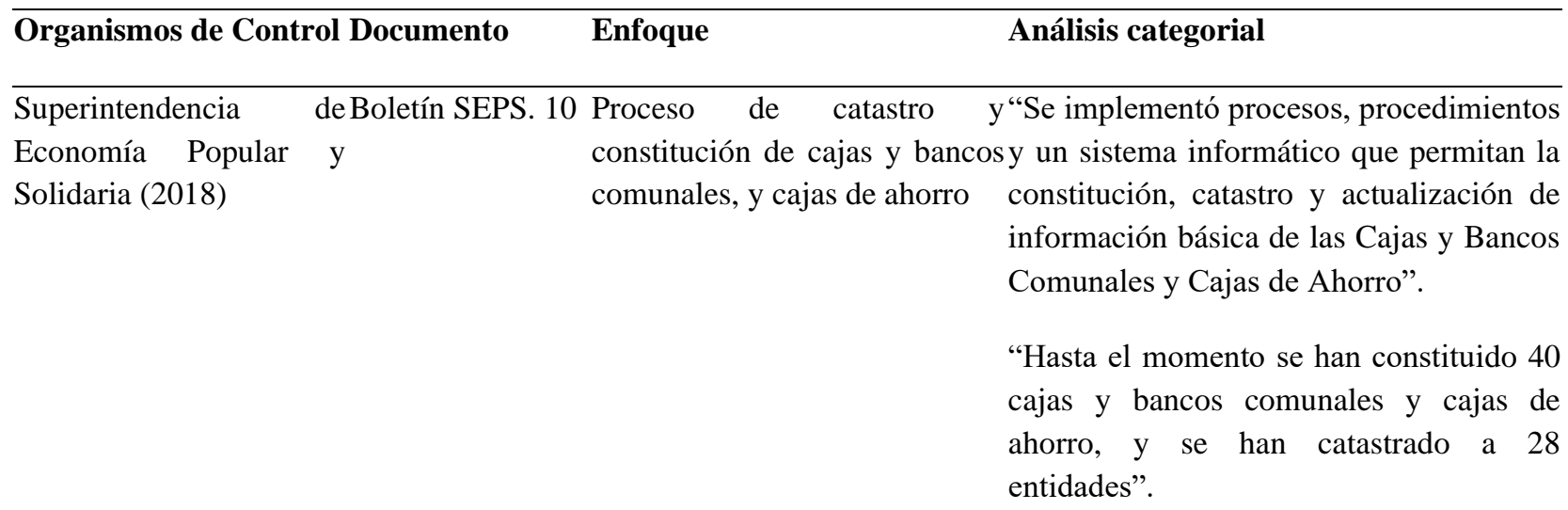

Superintendencia de Sala de prensa. Organizaciones del Oro son "Temas referentes al reglamento y la Ley Economía Popular y capacitadas por la SEPS. Orgánica de Economía Popular y Solidaria (2018) Noticia
En estas jornadas se capacito 17 personas de la caja de ahorro que tiene la Superintendencia, la estructura "Huaqsol" de Huaquillas. de las organizaciones y las buenas prácticas del Gobierno Cooperativo"

\begin{tabular}{|c|c|c|}
\hline Superintendencia & de OFICIO & permitidas, "Las cajas de ahorro son organizaciones \\
\hline Economía & y CIRCULAR & Nro.limitaciones y sanciones a lasque se forman er \\
\hline Solidaria (2019) & $\begin{array}{l}\text { SEPS-SGD } \\
2019-00903\end{array}$ & $\begin{array}{c}\text { Cajas que puedan captar fondos de terceros } \mathrm{u} \\
\text { otorgar créditos a terceros a quienes no }\end{array}$ \\
\hline
\end{tabular}




\begin{tabular}{|c|c|c|}
\hline \multirow{3}{*}{$\begin{array}{l}\text { Superintendencia } \\
\text { Economía Popular } \\
\text { Solidaria /2017) }\end{array}$} & de Sala de prensa. & $\begin{array}{l}\text { SEPS capacita a representantes "La } \\
\text { de Cajas y Bancos Comunales, Popular y }\end{array}$ \\
\hline & $\mathrm{y}$ & \\
\hline & & $\begin{array}{l}\text { miembros y representantes de cajas y } \\
\text { bancos comunales, y cajas de ahorro de } \\
\text { Portoviejo. Su objetivo es fortalecer a las } \\
\text { entidades de la economía popular y } \\
\text { solidaria, a través de un plan de } \\
\text { capacitación estructurado". }\end{array}$ \\
\hline
\end{tabular}

Junta de Regulación. Resolución No.Cajas de Ahorro Monetaria y Financiera436-2018-F

(2018)

\begin{abstract}
"Las entidades asociativas o solidaras, cajas y bancos comunales y cajas de ahorro son organizaciones que podrán optar por la personalidad jurídica, que se forman por voluntad de sus socios dentro del límite y en la forma determinada por la Junta de Política y Regulación Monetaria y Financiera, tendrán su propia estructura de gobierno, administración, representación, auto control social y rendición de cuentas y tendrán la obligación de remitir la información que /es sea solicitada por la superintendencia".
\end{abstract}

Elaboración propia a partir de la Bibliografía consultada

La normativa ecuatoriana ha establecido todos los parámetros para que las cajas de ahorros puedan desarrollarse en el ámbito financiero, es así que establece un Manual de Control donde podrán identificar la gestión tributaria, de acuerdo a los procedimientos de control interno que establezcan las mismas para el cumplimiento de sus obligaciones tributarias, por medio de auditorías con profesionales calificados por la Superintendencia de Economía Popular y Solidaria.

La revisión documental permitió establecer los puntos clave de la problemática lo cual se evidenció que las cajas de ahorros cuentan con normativas legales que permiten establecerse como una entidad consolidada y formal; sin embargo, sus estructuras internas no cuentan con todos los parámetros, es así que no presentan un modelo de gestión tributaria, en el cual lleven un control de todas las operaciones.

El proceso del cambio en las cajas de ahorro es lento debido al tiempo de adaptación y los nuevos requerimientos solicitados por la Superintendencia, hace que la manera de trabajar cambie y sea un proceso de innovación en cuanto a llevar metodologías financieras que contribuyan a la gestión tributaria de cada caja de ahorro constituida; sin embargo, estos pasos lentos influyen 
negativamente en la responsabilidad social que tiene dicha personería jurídica debido que descuidan las inversiones que deben realizar como obra social (Mayorga, Álvarez, \& Mayorga, 2018).

El modelo de gestión tributaria establecido por el Servicio de Rentas Internas, es un modelo relativamente básico (Figura 1) en el que solo se tiene un control de las organizaciones de economía popular y solidaria que forman parte del sistema financiero cooperativo (cajas de ahorro), debido que no cuentan con controles necesarios que permitan establecer datos reales en cuanto a su gestión, sin embargo, el modelo de brechas ha dado buenos resultados en estos últimos tiempos permitiendo establecer un orden en el proceso, teniendo mejores controles para todas las instituciones financieras, no solo para la caja de ahorro; es la más flexible en su aplicación ayudando a establecer o modificar parámetros que contribuyan al control de la gestión tributaria en estas organizaciones.

El modelo de consistencia, de insumo y de equilibro se han evidenciado que son una extensión del modelo de brechas, que se pueden combinar un modelo con otro para dar un mayor énfasis en el control; sin embargo, debido a que las cajas de ahorro son organizaciones pequeñas, estos modelos son más aplicados a las grandes instituciones financieras que requieren de una revisión mayor, con más problemas por solucionar.

El sistema de control interno se desenvuelve en las organizaciones como proceso de gestión para la toma de decisiones y cumpliendo los objetivos el control interno está basado en acciones, pronunciamientos y procedimientos el cual se basa en componentes como: Ambiente de control, evaluación de riesgos, actividades de control, informe y seguimiento, monitoreo. Los componentes del control interno identificados anteriormente a pesar de estar manifestado en la normativa jurídica no han sido diseñados o implantados con procedimientos específicos para las cajas de ahorros, lo que conlleva que estos sujetos no incorporen dentro de su gestión, componentes tales como manejos del medio ambiente, recursos humanos, indicadores financieros, recursos para manejar la información y comunicación hacia la comunidad, entre otros. (Mayorga, Álvarez, \& Mayorga, 2018)

Por consiguiente, el modelo de brechas propuesto por el Servicio de Rentas Internas como organismo de control es el que más se acerca a los requerimientos de la gestión en las cajas de ahorro; y puede combinarse con los componentes de control interno originando un informe de políticas que contendría la relación entre los componentes del control interno y el modelo de brechas, permitiendo que a través de la evaluación de un sistema de control interno bien estructurado se viabilice la gestión tributaria en las cajas de ahorro, en donde sea parte de la cultura organizacional para el cumplimiento apropiado de las políticas tributarias permitiendo de esta manera el desarrollo de un direccionamiento estratégico oportuno en estas organizaciones. (Figura $5)$. 
Vol. 4, $\mathrm{N}^{\circ}$ 2, p. 22-39, abril - junio, 2020

Figura 5. Procedimiento de control para viabilizar la gestión tributaria en cajas de ahorros

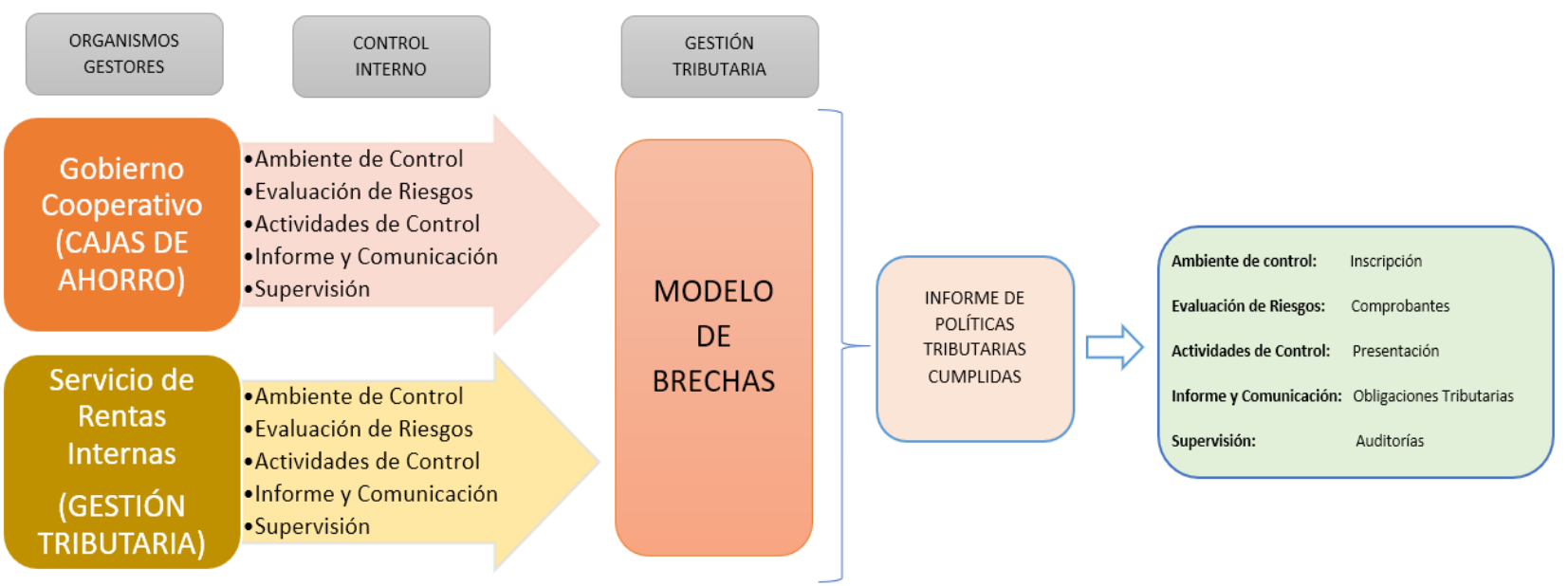

Elaboración propia

\section{Conclusiones.}

- Las cajas de ahorro realizan sus procesos individualmente de acuerdo a sus necesidades, es decir, no efectúan una planificación en la que se detallen indicadores de cumplimiento que coadyuven a mejorar las operaciones en cuanto a la gestión tributaria.

- Carencia de un manual de procesos administrativos, financieros y tributarios en las cajas de ahorro con relación a las obligaciones con el Servicio de Rentas Internas; en una forma más detallada que permita tener una orientación precisa para estas organizaciones, referente en la manera de registrar sus transacciones diarias y como otorgar los créditos a sus socios.

- Debido a las transiciones identificadas en la ley para las cajas de ahorro, se ha visto que las cajas de ahorros todavía deben consolidar sus estructuras internas, estableciendo procedimientos de control internos, de acuerdo al Modelo de Control que establece la Superintendencia de Economía Popular y Solidaria; que permitan cumplir con sus obligaciones tributarias donde puedan aplicar metodologías financieras que ayuden a tener un control de sus ingresos y egresos o ahorro - inversión.

- La correlación entre los componentes del modelo de Brechas y componentes del sistema de control interno, permite generar reportes para los organismos de control interno como externos de las Cajas de Ahorro, lo cual permitirá la toma de decisiones consensuadas.

\section{Referencias bibliográficas.}

Acuña, F. P. (2013). Control interno y su incidencia en la rentabilidad de la empresa cobros norte S.A. Revista Cientifica de FAREM - Estelí. Recuperado el 16 de 05 de 2019 
Barona, C. B. (2012). Políticas y Procedimientos de control Interno y Incidencia en la rentabilidad en la Cooperativa de Transporte Urbano Tungurahua. Recuperado el 12 de 06 de 2019, de http://repositorio.uta.edu.ec/bitstream/123456789/3098/1/TA0259.pdf

Barreix, A. \&. (2016). Un nuevo modelo de Administración Tributaria. Revista de la Facultad de Ciencias Económicas, (2).

Bermeo, M. J. (2016). Análisis documental de la V heuristica mediante la cartografía conceptual. RA XIMHAI, 12(6), 20. Recuperado el 22 de 09 de 2019, de https://www.redalyc.org/pdf/461/46148194006.pdf

Código Monetario y Financiero . (12 de 09 de 2014). Obtenido de Codigo Monetario y Financiero

Constituyente, A. . (2008). Obtenido de Constitución de la República del Ecuador.: https://s3.amazonaws.com/academia.edu.documents/45208547/constitucionecuador.pdf?AWSAccessKeyId=AKIAIWOWYYGZ2Y53UL3A\&Expires=1558766427 $\&$ Signature=RkIF5KrSIx37c\%2FKuaFx72jX5ynU\%3D\&response-contentdisposition=inline $\% 3 \mathrm{~B} \% 20$ filename\%3DConstitucion-ecuador.pd

Contadores Publicos de México. (2016). www.ccpm.org.mx. Recuperado el 14 de 05 de 2019, de https://www.ccpm.org.mx/avisos/el-control-interno-blog-dinero-en-imagen-agosto2016.pdf

Corporación Nacional de Finanzas Populares y Solidarias. (Ene - Feb de 2018). USD 5 millones para las cajas de ahorro y bancos comunales del Ecuador. Boletín SEPS 10, págs. 1-12. Obtenido de http://www.seps.gob.ec/documents/20181/455927/Boleti\%CC\%81n+SEPS+10_c.pdf/a79 0c288-d98c-405a-9f8f-f7799012deb4?version=1.0

Dirección Nacional de Planificación y Gestión Estratégica . (2016). Plan Estratégico Institucional 2016 - 2019. Quito: Servicios de Rentas Internas.

Encarna. (2016). Nuevo modelo de gestíon tributaria y de recaudación. Recuperado el 12 de 05 de 2019, de Nuevo modelo de gestíon tributaria y de recaudación: gestión de calidad y administracion electrónica al servicio de los ciudadanos y otras administraciones: https://www.google.com/search?client=firefox-b$\mathrm{d} \& \mathrm{q}=\mathrm{Nuevo}+$ modelo+de+gesti\%C3\%B3n+tributaria+y+de+recaudaci\%C3\%B3n\%3A+ Gesti\%C3\%B3n+de+calidad+y+administraci\%C3\%B3n+electr\%C3\%B3nica+al+servici o+de+los+ciudadanos $\% 2 \mathrm{C}+2016$

Escuela de Negocios y Dirección . (2015). Principios y Elementos de los Tributos. Recuperado el 24 de 05 de 2019, de Escuela de Negocios y su Implementacion: https://www.escueladenegociosydireccion.com/documentoscampus/FISCAL/LGT/NotaT ecnica/ENYDASESO1258.pdf 
Escuela Pública Digital. (2016). Legislación Laboral e Impositiva. Recuperado el 25 de 05 de 2019, http://contenidosdigitales.ulp.edu.ar/exe/legislacion/qu_es_la_obligacin_tributaria_y_sus _elementos.html

Hernández, M. (2018). Informe de Rendición de cuentas 2018. Quito: Superintendencia de Economía Popular y Solidaria.

Hora, M. d. (2019). Más de 117 cooperativas de ahorro y crédito en Tungurahua.

Ley de Regimen Tributario Interno. (21 de 10 de 2018).

Ley Orgánica de Economía Popular y Solidaria. (2019). Obtenido de Superintendencia de Economía Popular y Solidaria.

Mayorga. (2018). Auditoría de gestión con un enfoque de responsabilidad social. Quito, Pichicha , Ecuador : Editorial Jurídica del Ecuador. Recuperado el 18 de 05 de 2019

Mayorga-Díaz, M. P., Quezada-Sarmiento, P. A., Álvarez-Gómez, G., Alvarado-Camacho, P. E., López-Jara, A. A., \& Lopez-Paredes, A. (2018, June). Conceptual model and process applied in management auditic with focus in social responsibility through open source tools. In 2018 13th Iberian Conference on Information Systems and Technologies (CISTI) (pp. 1-6). IEEE.

Mayorga, M., Alvarez, G., \& Mayorga, L. (2018). Procedimiento para diagnosticar el componente social en la auditoria de gestión de cajas de ahorro. Revista de Ciencia, Tecnología e innvovación: Uniandes Episteme, 5(1), 66-76.

Morales, A. (2018). El sistema cooperativo de ahorro y crédito del Ecuadr a través de la historia. Revista Observatorio de la Economía Latinoamericana, 1-7.

Onrubia, J. (2016). Financiación autonómica y administración tributaria: UNa propuesta de modelo integrado de gestión. Recuperado el 25 de 05 de 2019, de https://www.fedea.net/financiacion-autonomica-y-administracion-tributaria-unapropuesta-de-modelo-integrado-de-gestion/

Plasencia, C. (2010). El sistema de Control Interno: Garantia del logro de los objetivos. Scielo, 14, 05. Recuperado el 14 de 05 de 2019

Quinaluisa, N., Ponce, V., Muñoz, S., Ortega, X., \& Pérez, J. (2018). El control interno y sus herramientas de aplicación entre COSO y COCO. Revista Cofin Habana, 12(1), 1.

Ramírez, J. (2010). Modelo de Macro-Consistencia Tributaria del Ecuador. Quito: Servicio de Rentas Interna.

Reglamento a la Ley Organica de Economia Popular y Solidaria . (27 de 02 de 2012). 
Reglamento a la Ley Orgánica Economía Popular y Solidaria. (22 de marzo de 2018). Decreto Ejecutivo 1061. Quito, Pichincha, Ecuador: Registro Oficial Asamblea Nacional.

Rodríguez D., T., Abella, Y., \& Pineda C., A. (2016). Revisión documental, una herramienta para el mejoramiento de las competencias de lectura y escritura en estudiantes universitarios. Chakiñan Revista de Ciencias Sociales y Humanidades, 46-56. Recuperado el 20 de 09 de 2019, de file:///C:/Users/Erika/Downloads/DialnetRevisionDocumentalUnaHerramientaParaElMejoramiento-6294862\%20(4).pdf

Saltos, J., Mayorga, M., \& Ruso, F. (2016). La economía popular y solidaria: un estudio exploratorio del sistema en Ecuador con enfoque de control y fiscalización. Revista Cofin Habana, 10(2), 1-3.

Serrano, J. C. (2018). Análisis Dinámico de la Recaudación Tributaria en el Ecuador Aplicando Modelos Var. Quito: Servicio de Rentas Internas (SRI).

Servicio de Rentas Internas. (2018). Obtenido de www.sri.gob.ec

Servicio de Rentas Internas. (23 de 10 de 2018). Código Tributario Interno. Quito. Recuperado el 22 de 05 de 2019

Superintendencia de Economía Popular y Solidaria. (2019). Ecuador tiene un total de 887 cooperativas de ahorro y crédito. Quito, Pichincha, Ecuador.

Valencia, V. (2015). Revision documental en el proceso de investigación. Universidad Tecnológica de Pereira. Recuperado el 25 de 08 de 2019, de https://univirtual.utp.edu.co/pandora/recursos/1000/1771/1771.pdf

Vargas, E. (2016). La recaudación tributaria y su relación con la gestion administrativa en la municipalidad distrital de Taricá. Recuperado el 23 de 05 de 2019, de http://repositorio.uladech.edu.pe/bitstream/handle/123456789/998/RECAUDACION_TR IBUTARIA_TASAS_DELGADO_VARGAS_EDITA_MAGALI.pdf?sequence=4\&isAll owed $=\mathrm{y}$

Vega de la Cruz, \&. N. (2016). Procedimiento para la gestión de la Supervición y Monitoreo del Control Interno. Ciencias Holguín, 20. Recuperado el 20 de 05 de 2019, de https://www.redalyc.org/pdf/1815/181543577007.pdf

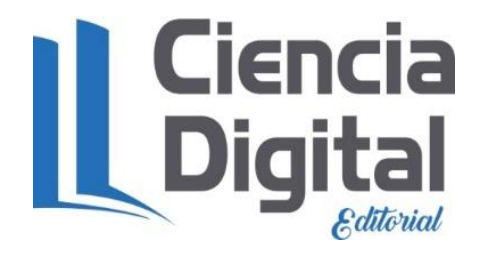


PARA CITAR EL ARTÍCULO INDEXADO.

Mayorga Díaz, M. P., Leguisamo Rosado , E. V., López Paredes, M. A., \& Espinosa Jaramillo, M. T. (2020). La gestión tributaria en cajas de ahorros como herramienta de control. Visionario Digital, 4(2), 22-39. https://doi.org/10.33262/visionariodigital.v4i2.1213

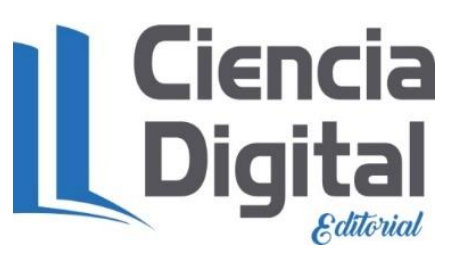

El artículo que se publica es de exclusiva responsabilidad de los autores y no necesariamente reflejan el pensamiento de la Revista Visionario Digital.

El artículo queda en propiedad de la revista y, por tanto, su publicación parcial y/o total en otro medio tiene que ser autorizado por el director de la Revista Visionario Digital.
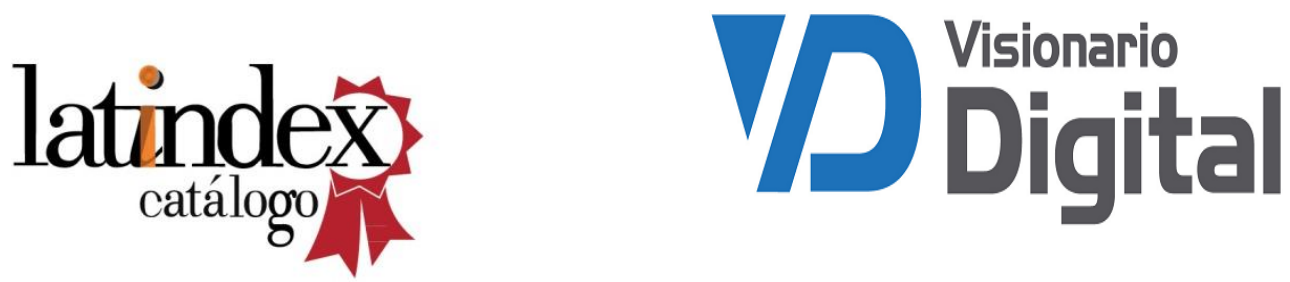